\title{
Bronchial Artery Embolization, an Increasingly Used Method for Hemoptysis; Treatment and Avoidance
}

\author{
(1) Ugur Temel, ${ }^{1}$ () Asli Gul Akgul, ${ }^{1}$ ㄴ) Sevtap Dogan' \\ 'Department of Thoracic Surgery, University of Health Sciences Turkey, Sisli Hamidiye Etfal Teaching and Resarch Hospital, Istanbul, Turkey \\ ${ }^{2}$ Department of Radiology, Kocaeli University Faculty of Medicine, Kocaeli, Turkey
}

\begin{abstract}
Objectives: Hemoptysis is an alarming symptom. It may cause some severe life-threatening complications. Hypertrophic and fragile bronchial artery causes hemoptysis and occurs mostly in bronchiectasis, sarcoidosis, active or sequelae tuberculosis, aspergilloma, lung cancer or cystic fibrosis. Bronchial artery embolization is one of the angiographic methods used in diagnosis and treatment for years performed by radiologists. Hemoptysis is used mostly in patients with hemoptysis. Using this method, surgical management with high mortality and morbidity rates can be avoided or better conditions for surgery can be provided via stopping hemorrhage before surgery. We aim to share the experiences of our hospital about patients who underwent bronchial artery embolization and compare our results with the literature.

Methods: Thirty-nine patients (29 male, 10 female) underwent angiography-aiming embolization. Pathologies were hemoptysis in 37 patients, Castleman disease in two patients. Embolization was performed in 33 patients; 31 for hemoptysis, two for Castleman disease. Bilateral embolization was performed in six patients.

Results: Computed tomography (CT) was helpful in diagnosing the side of bleeding in $91.8 \%$ of the patients with hemoptysis. Bronchoscopy was diagnostic in $53 \%$ of patients. Polyvinyl alcohol $(n=27)$ was mostly used for embolization. Hemoptysis recurred in six patients (19.3\%). All were managed successfully, of four with re-embolization. One major complication, transient blindness, was observed.

Conclusion: Bronchial artery embolization is minimally invasive, more tolerable compared to surgery can be managed with high success and lower complication rates, especially hemoptysis and in some other situations. It provides time for evaluating the underlying disease and delaying surgery for elective conditions. That is why this method has been used increasingly.

Keywords: Bronchial artery; embolization; hemoptysis; interventional angiography.

Please cite this article as "Temel U, Akgul AG, Dogan S. Bronchial Artery Embolization, an Increasingly Used Method for Hemoptysis; Treatment and Avoidance. Med Bull Sisli Etfal Hosp 2020;54(3):313-319". ifferent angiographic methods have been used in diagnosis and treatment for years. Bronchial artery embolization (BAE) is a minimally invasive therapeutic intervention. The most important indication of $B A E$ is massive or moderate hemoptysis. Lungs have dual blood supply; bronchial and pulmonary arteries but bronchial system

are responsible for almost $90 \%$ of hemoptysis. Hypertrophic bronchial arteries and abnormal bronchopulmonary shunts occur in chronically inflamed lung tissue and due to increased vessel's fragility, inflammation causes rupture of vessels and bleeding. The aim of BAE is the occlusion of these vessels selectively. ${ }^{[1]}$
\end{abstract}

$\mathrm{D}$

Address for correspondence: Asli Gul Akgul, MD. Saglik Bilimleri Universitesi Sisli Etfal Egitim ve Arastirma Hastanesi Gogus Cerrahisi Anabilim Dali Tip Fakultesi, Istanbul, Turkey

Phone: +90 5064971969 E-mail: asliakgul@gmail.com

Submitted Date: May 06, 2020 Accepted Date: June 16, 2020 Available Online Date: August 25, 2020

${ }^{\circ}$ Copyright 2020 by The Medical Bulletin of Sisli Etfal Hospital - Available online at www.sislietfaltip.org

OPEN ACCESS This is an open access article under the CC BY-NC license (http://creativecommons.org/licenses/by-nc/4.0/). 
Hypertrophic and fragile bronchial arteries occur mostly in bronchiectasis, sarcoidosis, active or sequelae tuberculosis, aspergilloma, lung cancer or cystic fibrosis. ${ }^{[2,3]}$ In some of the cases, the definitive diagnosis cannot be reached (3$42 \%)^{[4,5]}$ Some other system pathologies and cardiovascular causes as mitral stenosis must also be kept in mind in this idiopathic group. Surgery always may have a high risk of morbidity and mortality in those conditions. Additionally, surgery may cure focal diseases, but in diffuse ones, it may be riskier because of the decreased pulmonary reserve. The mortality rate is reported between $50-100 \%$ in conservatively treated hemoptysis. ${ }^{[1]}$ The mortality rate of the surgical treated massive hemoptysis is pointed out between $7-40 \%{ }^{[2-6]}$

We have some techniques routinely used in hemoptysis for diagnosis or treatment. Besides, surgery, mostly rigid and in mild hemorrhages fiberoptic bronchoscopy, is used for many years. In mild to moderate, or massive hemoptysis, bronchoscopy was used mostly in the theatre room with both aims of localisation and treatment. Thus, it could be easy to turn surgery quickly if the endobronchial methods like coagulation with specific agents, cauterisation, cryogenic procedures, or blockage of the bleeding part with specific materials would be ineffective.

Bronchial artery embolization prevents urgent surgery, and in some cases, provides more appropriate conditions for surgical interventions in hemoptysis. Additionally, in Castleman disease, BAE may be used preoperatively or for inoperable diseases because of the high risk of bleeding. ${ }^{[7,}$ ${ }^{8]}$ Here, we aimed to share and compare our experiences in bronchial artery embolization.

\section{Methods}

In our hospital, 39 patients underwent angiography with the aim of embolization during a 5-years period. Patients' data were evaluated retrospectively, depending upon their hospital files. The Ethics Committee of our institution approved this study.

There were 29 male and 10 female patients. The median age was 53 (min: 11- max: 77). Angiography was performed in 37 patients with hemoptysis in two patients aiming to close the feeding vessel of masses. The mostly seen etiologic factor of hemoptysis was active tuberculosis or tuberculosis sequel in this series $(n=16)$. One of the patients with tuberculosis had a history of lobectomy. Bronchiectasis was the etiologic factor in eight patients. Others were summarized in Table 1. In two patients with Castleman disease, the blockage of the feeding vessels of the masses was aimed because of extreme vascularity (Table 1 ).

All patients were evaluated using chest $\mathrm{x}$-rays and thorax
Table 1. Etiologies for bronchial artery angiography with or without embolization

\begin{tabular}{lc}
\hline Causes of Angiography & Number of patients \\
\hline Active/Sequel Tuberculosis & 16 \\
Bronchiectasis & 8 \\
Chronic Obstructive Pulmonary Disease & 4 \\
Non-specific sequel & 3 \\
No lung pathology & 3 \\
Castleman & 2 \\
Operated hydatid cyst & 1 \\
Inoperable lung cancer & 1 \\
Congenital vascular abnormality & 1 \\
Total & 39 \\
\hline
\end{tabular}

CTs before the angiography procedure. In 13 patients, rigid or fiberoptic bronchoscopy was performed. All patients underwent angiography, but embolization was performed in 33 patients. Angiography and embolization are performed under local anaesthesia using common femoral artery access via five or four French vascular sheaths. Polyvinyl alcohol (PVA) was used in 27 patients (Figs. 1, 2), histoacryl/

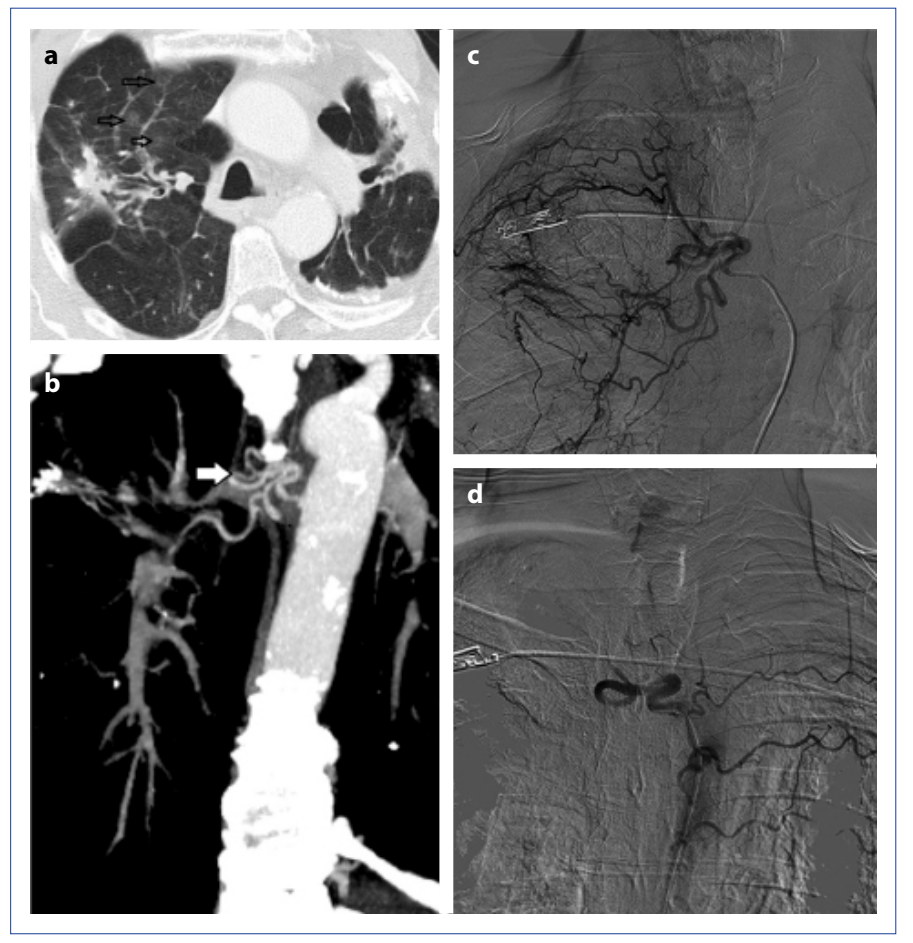

Figure 1. Patient with hemoptysis due to bronchiectasis. Axial section CT image (a) shows bilaterally bronchiectasis, sequels and right upper lobe focal ground-glass opacity because of alveolar hemorrhage. Coronal reconstruction section CT image (b) shows the right abnormal bronchial vessels. In angiography (c), dilatation, hypertrophy and abnormal vascularity at the intercostal branch and bronchial artery originating from right intercostobrachial truncus. Totally obstruction at the right intercostobrachial truncus after PVA embolization (d). Left intercostal branches are visible due to the reflux of contrast. 


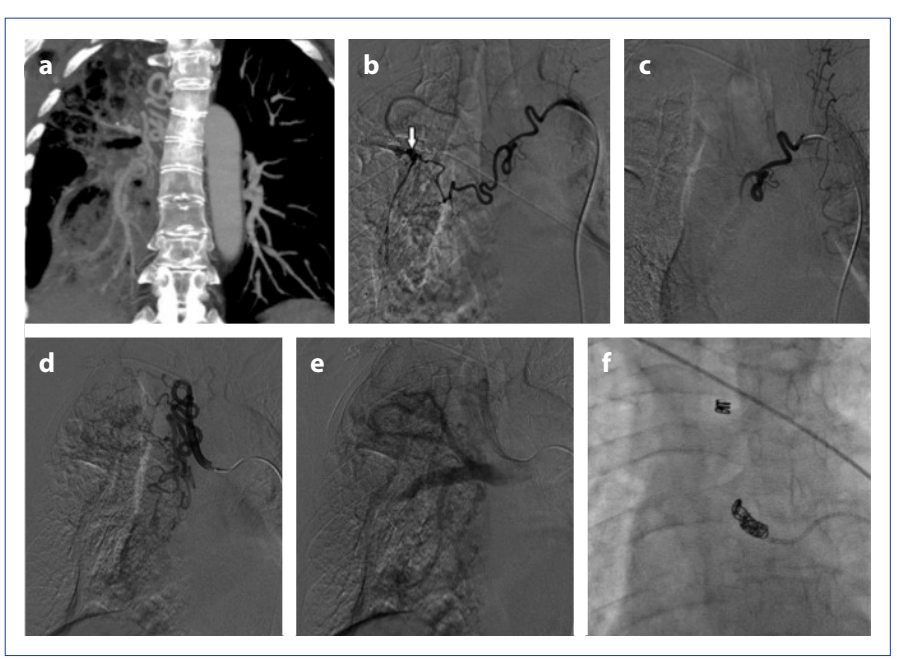

Figure 2. Coronal oblique CT reconstruction (a) shows pulmonary bronchitis sequels and the right abnormal bronchial vascular system. Descending aorta and normal pulmonary arteries are obvious at the left side. In angiography (b), dilatation, abnormal hypervascularity and aneurysmatic enlargement at right bronchial artery. Angiography shows (c) the first bronchial artery was totally occluded with PVA. Tortiousity and dilatation at right second bronchial artery (d). Abnormal hypervascularity and neovascularization at the feeding area. In late angiographic images (e) demonstrates fistulisation between this artery and pulmonary system. Coil embolization was used for BAE ( $f$ ) because of the fistulization to the pulmonary system and having a large size in the diameter of the bronchial artery.

lipiodol in two (Fig. 3), PVA and mechanical coil in one, PVA and cyanoacrylate in one patient. Coil embolization alone is used in 1 patient and cyanoacrylate was used in one patient. Angiographic images were carefully evaluated to determine the anatomy of bronchial arteries and to establish the hypertrophic arteries to perform BAE. Descending thoracic aortogram and selective catheterization of bronchial arteries are generally performed with cobra-type

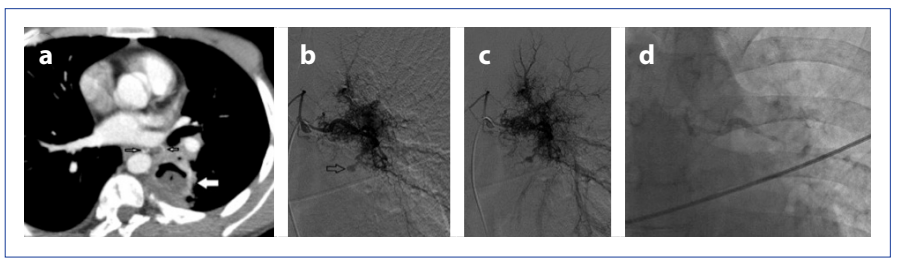

Figure 3. Axial CT image shows left lower lobe cavitary lesion compatible with a thick wall abscess. An abnormal dilated bronchial artery adjacent to the cavity. In angiographic images (b) demonstrate right and left bronchial arteries originate from the same truncus. Dense pathological vascular system arising from the left bronchial artery is obvious. Left bronchial artery is markedly tortuous. A small aneurysmatic enlargement exists in this pathologic region. Fistulization at late images (c) was seen between the bronchial artery and pulmonary vascular system. In angiography (d) abnormal left bronchial artery occlusion after histoacryl embolization was observed. curved catheters. Hypertrophy, enlargement and tortuosity of bronchial arteries, neovascularity, hypervascularity, arterio-venous shunts, contrast medium extravasation and aneurysms are abnormalities that can be observed in angiograms. After selective catheterization of the abnormal and enlarged bronchial artery, the embolic material is injected with small-volume syringes by hand injection method under fluoroscopic real-time imaging to avoid reflux. We preferred to use particles between 350-500 microns in diameter in all patients because small size particles could cause very distal embolization occluding the end organs, such as bronchi, esophagus, or vascular structures, at the precapillary level, which may result in ischemic complications. The median follow-up period was 22 months (range between 1-81 months).

\section{Results}

Computed tomography was helpful in 36 of the 39 patients $(92.3 \%)$ in determining the etiology. For hemoptysis, CT showed the bleeding area in 34 of 39 patients (91.8\%). In 13 patients, bronchoscopy was performed before angiography; the active bleeding area was localized in seven patients (53\%). In 20 patients, lung pathology was determined on the right side, left in 13, and in six patients bilaterally.

All patients underwent angiography, but for 33 of them, embolization was performed. In six patients whose embolization did not perform, three of the cases were tuberculosis, two were chronic obstructive pulmonary disease (COPD), and one was bronchiectasis. In three cases, it was not able to determine the bleeding vessels selectively. In two patients, hemoptysis was discontinued spontaneously, so the bleeding focus could not be localized. In one patient, the bleeding site was localized, but because of the extremely thin vessels, embolization could not be performed. In two cases whose hemoptysis discontinued spontaneously, hemoptysis recurred in one week and three months, respectively. Embolization was performed successfully in that period of time in those patients. Bilateral embolization in the same session was performed in three patients with tuberculosis and in three patients with non-specific sequels.

Hemoptysis recurred in six patients after BAE $(6 / 31 ; 19.3 \%)$. In four of them, re-embolization was performed. One of the six patients was admitted with recurrent hemoptysis after nine months follow up period, and embolization was required to be performed for the opposite side. This patient had inoperable diffuse bronchiectasis. Second embolization was also required in three patients; one with tuberculosis, one with vascular anomaly and one with vasculitis. Recurrence time was three months, one month and five years, respectively. The patient with tuberculosis was admitted to 
hospital with a third attack of hemoptysis after one month from the second embolization and this case was treated conservatively. In one patient with recurrent hemoptysis (in 9-month-follow-up), conservative management was successful and no embolization was needed. In four patients of 27 in whom PVA was used for hemoptysis, recurrence was seen (14.8\%).

In two cases with Castleman's disease, BAE was also performed for extreme vascularity of the lesion. In one patient, thorax $C T$ revealed its increased vascularity of the lesion and BAE was performed before the operation for decreasing the risk of major bleeding in operation. Embolization was performed to the other patient after surgery. Total resection of the mass could not be achieved in operation and after surgery, BAE was performed, aiming to reduce the mass size and decrease the risk of bleeding (Fig. 4). Finally, in the median follow up period of 38 months, all the patients included recurrent ones, too, were free of any symp-
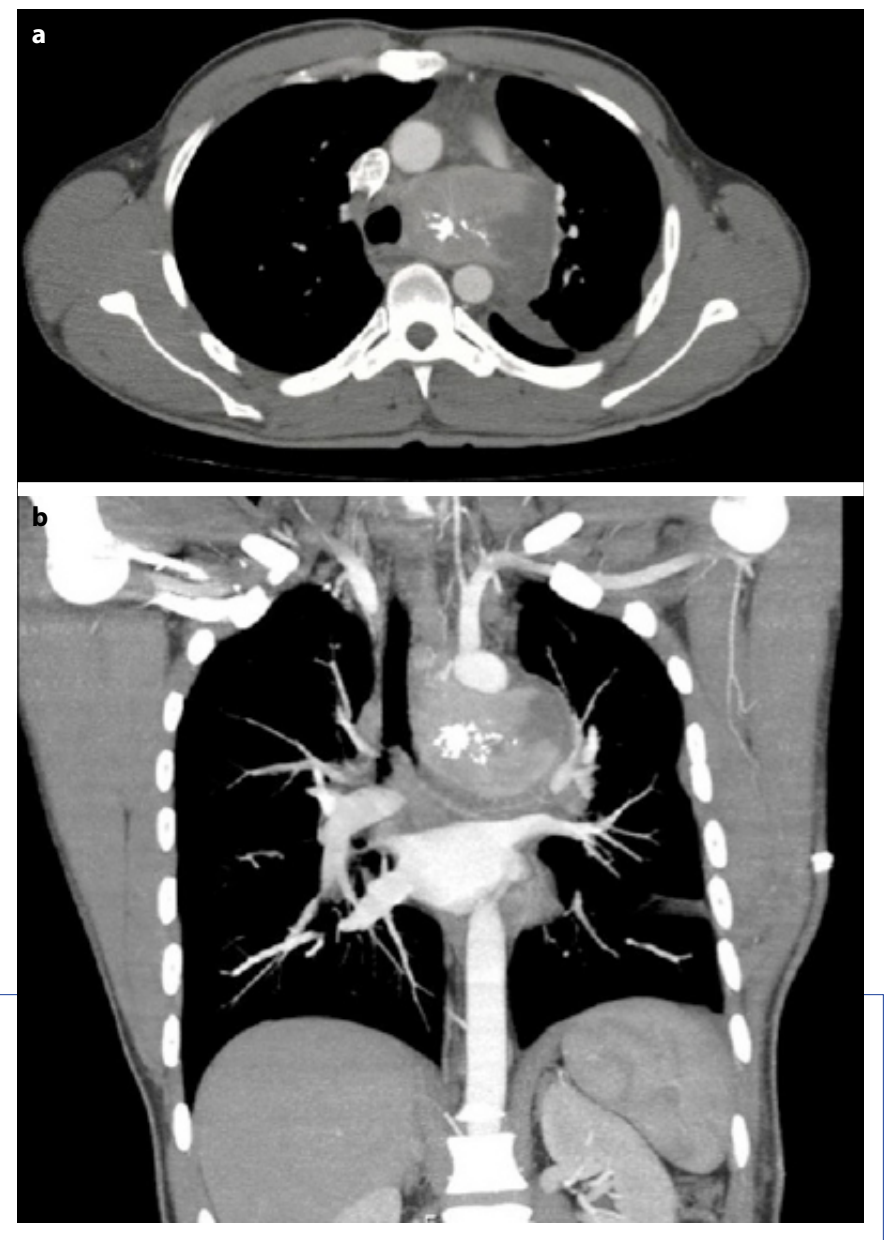

Figure 4. In the axial section of CT examination (a) shows nonhomogenous calcified mass filling aortapulmonary window. The coronal section of CT examination (b) demonstrates mass compression to the adjacent structures. toms. Besides, two patients with Castleman disease, the rest of the patients had diffuse lesions or were medically inoperative. There was no need for surgery, or any other intervention.

The most common complaint after the procedure was chest pain with varying degrees. In one patient, temporary loss of vision thought to occur due to the occlusion of the ophthalmic artery was detected. The patient regained his normal visual functions after hyperbaric oxygen therapy. There was no other complication. During the follow-up period of 22 months' median time, five patients were admitted with recurrent hemoptysis between 1-60 months.

\section{Discussion}

Hemoptysis is an alarming symptom. It may cause some severe life-threatening complications. Bleeding may compromise the airway causing asphyxia and death. Although lungs have a dual blood system, bronchial arteries are responsible for $90 \%$ of all hemoptysis. ${ }^{[9]}$ The bronchial arteries originate from the aorta, and they can be imaged with angiographically. Their embolization can be performed using various agents to stop bleeding. The first report of bronchial artery embolization for hemoptysis was published in 1973 by Remy ${ }^{[10]}$ In the treatment of hemoptysis, BAE is getting to use more frequently nowadays. Using this method, high mortality and morbidity rates can be avoided.

Reduced pulmonary circulation and hypoxic vasoconstriction, intravascular thrombosis and vasculitis cause arteriolar occlusion in acute or chronic lung disease. Bronchial artery proliferates for compensation, and consequently, any infection or inflammation may lead to the rupture of the arteries. [9, 11] Most commonly, tuberculosis, bronchiectasis, cystic fibrosis, chronic inflammatory lung disease, aspergillosis, lung abscess, pneumonia, chronic bronchitis, pulmonary interstitial fibrosis, pneumoconiosis, pulmonary artery aneurysms may lead hemoptysis. Among the many different causes of hemoptysis, the most frequent worldwide is tuberculosis. In the western world, the cause of half the cases of hemoptysis remains unestablished. In the other half, the causative factors are inflammatory diseases of the airways $(25.8 \%)$, including tuberculosis (2.7\%) and aspergillosis (1.1\%), bronchial carcinoma and metastases, bronchiectasis, cardiovascular causes as mitral stenosis, pulmonary artery embolism, and anticoagulation treatment in order. ${ }^{[12]}$ In a recent study from Korea, hemoptysis in non-tuberculosis mycobacterial lung diseases of 183 patients was investigated. Those patients commonly experienced hemoptysis without specific risk factors. The success rate of BAE was high, and there were no severe complications associated. The authors indicate that the subjects in this study had a higher prevalence of pulmonary tubercu- 
losis history compared with other studies because of Korea having a high prevalence of tuberculosis. ${ }^{[13]}$ In our series, active tuberculosis or tuberculosis sequelae was determined in 16 patients.

Chest x-ray, CT and bronchoscopy are the most commonly performed diagnostic tools to locate the site of bleeding. However, in massive hemoptysis, those procedures cannot be performed because of the limited time. ${ }^{[9,14]}$ Contrastenhanced CT can localize the localization of hemoptysis at a rate of $63-100 \% .{ }^{[9]}$ We could perform chest $x$-ray and CT in all patients. Thorax $\mathrm{CT}$ revealed pathological findings of the probable causes for hemoptysis in $91.8 \%$ patients. Hsiao et al. ${ }^{[14]}$ reported that bronchoscopy was unnecessary before the BAE procedure. We performed bronchoscopy in 13 of our patients and the origin of the hemoptysis was detected in seven (53\%) patients.

The bronchial arteries arise from the descending thoracic aorta, commonly between the levels of the T5 and T6 vertebrae. They supply not only the airways and interstitium of the lung, but bronchovascular bundles, nerves, regional lymph nodes, visceral pleura, esophagus, vasa vasorum of the aorta, pulmonary artery, and pulmonary veins as well. [11, 15] Normal bronchial arteries measure $1.5 \mathrm{~mm}$ in diameter in adults. A bronchial artery larger than $3 \mathrm{~mm}$ is most likely considered abnormal. ${ }^{[11]}$ It is suggested that to combine $\mathrm{CT}$ images with angiographic findings will increase the sensitivity and specificity of the exact localization of hemoptysis. ${ }^{[16]}$

In our series, two cases with Castleman disease underwent angiography and BAE because of their high vascularity. In the literature, it was also pointed out successful embolization. ${ }^{[7,8]}$ One of our case embolization was performed before the operation for avoiding bleeding in operation, the other was inoperable in surgery, embolization was performed to avoid its complications (bleeding, compression adjacent structures).

Cobra-type curved, as we used, or Simmons-1 and headhunter catheters can be used for descending thoracic aortogram and selective catheterization of bronchial arteries. Different embolic materials can be used for BAE. Polyvinyl alcohol (PVA) particles are non-absorbable embolic materials that can be easily handled with available varying particle diameters and most frequently used worldwide. They do not undergo spontaneous absorption and therefore, can establish a more stable vascular occlusion. Embolization with PVA particles larger than $500 \mu \mathrm{m}$ causes occlusion of the very proximal part of the bronchial arteries and may produce a suboptimal result due to the tendency to form collaterals around the occlusion site. Utilizing particles smaller than $325 \mu \mathrm{m}$, can cause undesirable pass through the bronchopulmonary anastomosis, which was shown to reach approximately $325 \mu \mathrm{m}$ in human lung. ${ }^{[17-}$ 19] Utilization of PVA particles with a diameter of 350-500 $\mu \mathrm{m}$ is recommended to avoid complications; just we used in our patients. Liquid embolic agents are available but are not recommended to use because of the high risk of tissue necrosis. ${ }^{[4,9]}$

Stainless steel platinum coils are also generally not used for BAE because coil embolization results in undesired proximal occlusion that may result in re-bleeding. Gelfoam pledgets $(1-2 \mathrm{~mm})$, absorbable gelatine sponge, thrombin and glue can be used as embolization agents. ${ }^{[3]}$ Proximal embolization is not preferred because of rapid formation of distal arterial collaterals. If the embolization agent passes through the shunt site, it may cause parenchymal ischemia and necrosis.

In a study analyzing 10 patients who underwent BAE, PVA microspheres were used in eight of the BAE patients and mechanical coils used in the other two patients. Hemoptysis recurred in two patients that had been treated with mechanical coils. There were no recurrences among patients treated with PVA. ${ }^{[17]}$ Similarly, in another study, BAE was performed to five patients successfully for the treatment of massive hemoptysis due to pulmonary tuberculosis. ${ }^{[20]}$ We used 350-500 micron PVA particles in this study. In hemoptysis with PVA, the recurrence rate was $14.8 \%$ in our series. We mostly used PVA because of cost and safety. In cases that we could not have a suitable size, we preferred another agent. Coils were preferred in case of recurrence. Liquid agents were used for congenital vascular malformations and aneurysms.

Chest pain is the most common complication after BAE and usually self-limiting within 2-7 days. Transient dysphagia results from unintentional embolization of esophagus. Subintimal dissection of the aorta, the wrong target embolization, pulmonary infarction are the other possible complications. $[9,21,22]$ The most serious complication associated with BAE is spinal cord ischemia due to the inadvertent embolization of spinal arteries. The prevalence is reported to be $1.4-6.5 \% .23$ Another rare neurologic complication is the blindness that may result from unintentional embolization of the occipital cortex or directly ophthalmic artery in the setting of a fistula formation presents from the bronchial artery to the pulmonary veins. Spinal and bronchial arteries may originate from the same trunks. 10 In 5-10\% of cases, the major supply of the anterior spinal artery arises from a right intercostobrachial trunk. ${ }^{[9]}$ Therefore, it will be useful to perform a neurological examination before and after the procedure.

The most frequent complaint was chest pain varying in intensity in our patients after the procedure. Temporary loss 
of vision due to the occlusion of the ophthalmic artery was determined in one patient, and the patient regained his normal visual function after hyperbaric oxygen therapy. There was not any other complication.

Spinal arteries need special attention. The great anterior medullary artery, or the artery of Adamkiewicz, is a vessel that usually arises between T9 and T12 vertebral levels. It has a classical "hairpin" configuration at angiography. Embolization of this artery may cause spinal cord ischemia. Radicular arteries are more often seen on angiograms and presence of them is not considered to be a contraindication for embolization.9 Spinal, especially radicular arteries were also seen in our series (10\%), but Adamkiewicz was not seen. This may be because of our study protocol. We initially got fluoroscopic images; if needed, it is followed by angiography. Adamkiewicz also could not be seen due to the low dose of contrast with suboptimal imaging.

The shortcoming for this paper is the number of patients. As a result, BAE is a minimally invasive, well-tolerated method performed under local anesthesia, used in different clinical situations, including mostly hemoptysis. Furthermore, BAE has a high success rate and low complication rate. Bronchial artery embolization is a time-saving procedure for preventing patients from urgent surgical interventions; also allows researching and diagnosing the underlying disease. For these reasons, although it is an increasingly preferred and successfully applied technique, recurrence risk in the long-term follow-up and elective surgery for the main disease must also be kept in mind. Since the embolisation technique was started to perform in our hospital, this gives a chance to protect us from the mortality rates of urgent surgery in hemoptysis.

\section{Disclosures}

Ethics Committee Approval: Because of being an old investigation and report before the year 2020, we do not have any ethic form from an ethic committee. There is no name, face, or any spesific sign that indicates a person, or bring any name to mind. Presented at Toraks $16^{\text {th }}$ Congress at 2013, Antalya.

Peer-review: Externally peer-reviewed.

Conflict of Interest: None declared.

Authorship Contributions: Concept - A.G.A.; Design - U.T., S.D., A.G.A.; Supervision - S.D.; Materials - A.G.A., S.D.; Data collection \&/or processing - U.T., A.G.A.; Analysis and/or interpretation U.T.; Literature search - U.T.; Writing - U.T., A.G.A.; Critical review - S.D., A.G.A.,U.T.

\section{References}

1. Cauldwell EW, Siekert RG, Lininger RE, Anson BJ. The bronchial arteries. An anatomic study of 150 human cadavers. Surg Gynecol Obstet 1948;86:395-412.
2. Najarian KE, Morris CS. Arterial embolization in the chest. J Thorac Imaging 1998;13:93-104. [CrossRef]

3. Marshall TJ, Jackson JE. Vascular intervention in the thorax: bronchial artery embolization for haemoptysis. Eur Radiol 1997;7:1221-7. [CrossRef]

4. Burke CT, Mauro MA. Bronchial artery embolization. Semin Intervent Radiol 2004;21:43-8. [CrossRef]

5. Cornalba GP, Vella A, Barbosa F, Greco G, Michelozzi C, Sacrini A, et al. Bronchial and nonbronchial systemic artery embolization in managing haemoptysis: 31 years of experience. Radiol Med 2013;118:1171-83. [CrossRef]

6. Fernando HC, Stein M, Benfield JR, Link DP. Role of bronchial artery embolization in the management of hemoptysis. Arch Surg 1998;133:862-6. [CrossRef]

7. Lorenz JM, Zangan SM, Leef JA. Mediastinal Castleman disease: embolization without surgery. J Vasc Interv Radiol 2009;20:1393-4.

8. Safford SD, Lagoo AS, Mahaffey SA. Preoperative embolization as an adjunct to the operative management of mediastinal Castleman disease. J Pediatr Surg 2003;38:E21-3. [CrossRef]

9. Yoon W, Kim JK, Kim YH, Chung TW, Kang HK. Bronchial and nonbronchial systemic artery embolization for life-threatening hemoptysis: a comprehensive review. Radiographics 2002;22:1395409. [CrossRef]

10. Remy J, Voisin C, Ribet $M$, Dupuis C, Beguery $P$, Tonnel AB, et al. Treatment, by embolization, of severe or repeated hemoptysis associated with systemic hypervascularization. Nouv Presse Med 1973;2:2060.

11. Deffebach ME, Charan NB, Lakshminarayan S, Butler J. The bronchial circulation. Small, but a vital attribute of the lung. Am Rev Respir Dis 1987;135:463-81.

12. Ittrich $H$, Bockhorn $M, K$ Klose $H$, Simon $M$. The Diagnosis and Treatment of Hemoptysis. Dtsch Arztebl Int 2017;114:371-81. [CrossRef]

13. Lee SH, Lee JH, Chang JH, Kim SJ, Yoon HY, Shim SS, et al. Hemoptysis requiring bronchial artery embolization in patients with nontuberculous mycobacterial lung disease. BMC Pulm Med 2019;19:117. [CrossRef]

14. Hsiao El, Kirsch CM, Kagawa FT, Wehner JH, Jensen WA, Baxter RB. Utility of fiberoptic bronchoscopy before bronchial artery embolization for massive hemoptysis. AJR Am J Roentgenol 2001;177:861-7. [CrossRef]

15. Marshall TJ, Jackson JE. Vascular intervention in the thorax: bronchial artery embolization for haemoptysis. Eur Radiol 1997;7:1221-7. [CrossRef]

16. Sopko DR, Smith TP. Bronchial artery embolization for hemoptysis. Semin Intervent Radiol 2011;28:48-62. [CrossRef]

17. Ustünsöz B, Bozlar U, Kocaoğlu M, llica T, Dakak M. Mechanical coil embolization of pulmonary arteriovenous malformations. Diagn Interv Radiol 2006;12:39-42.

18. Pump KK. Distribution of bronchial arteries in the human lung. Chest 1972;62:447-51. [CrossRef] 
19. Çalışkan KC, Ulusay SM, Uysal E, Türk Ş, Karpat Z. Trans arterial microcatheter embolization treatment for bleeding bronchial artery branch. Med Bull Sisli Etfal Hosp 2010:44;120-3.

20. Carim I, Carim H, Modi M. Bronchial artery embolisation for the treatment of massive haemoptysis resulting from pulmonary tuberculosis. South African Journal of Radiology 2007;11:8-12.

21. Poyanlı A, Sencer S, Rozanes I, Acunaş B. An Effective Alternative in the Palliative Treatment of Massive Hemoptysis: Endovascular Intervention. Toraks 2001;2:22-6.

22. Mal H, Rullon I, Mellot F, Brugière O, Sleiman C, Menu Y, et al. Immediate and long-term results of bronchial artery embolization for life-threatening hemoptysis. Chest 1999;115:996-1001. [CrossRef]

23. Wong ML, Szkup P, Hopley MJ. Percutaneous embolotherapy for life-threatening hemoptysis. Chest 2002;121:95-102. [CrossRef] 